\title{
Desenvolvimento do algodoeiro em resposta a modo de aplicação e doses de cloreto de mepiquat via sementes
}

\author{
Development of cotton in response to mode of application and doses of mepiquat chloride in seeds
}

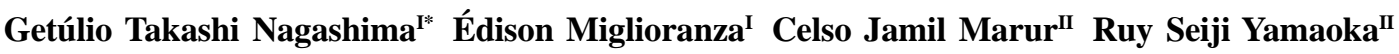 \\ João Guilherme dos Reis Silva ${ }^{\text {III }}$
}

\section{RESUMO}

A aplicação do regulador de crescimento via embebição de sementes apresenta alguns inconvenientes, dentre eles, a necessidade de secagem das sementes após o tratamento. O objetivo do trabalho foi avaliar o efeito do tratamento de sementes com Cloreto de Mepiquat (CM), embebidas $e$ aplicadas diretamente nas sementes de algodão, no crescimento das plantas. Os tratamentos foram arranjados em blocos casualizados, com seis repetições, utilizando a linhagem $P R$ 02-307. O experimento foi constituído por cinco tratamentos: sementes sem tratamento; aplicação direta sobre as sementes de CM a 3,75 e 7,5g i.a. $\mathrm{kg}^{-1}$ de sementes e embebidas em solução de CM a 3,75 e 7,5g i.a. $\mathrm{kg}^{-1}$ de sementes. Após os tratamentos, a semeadura foi feita em vasos com três litros de solo em casa de vegetação. Foram determinadas a altura e a área foliar do início até 90 dias após emergência e a fitomassa da matéria seca no final do experimento. Os tratamentos em que os reguladores de crescimento independentes da dose e forma de aplicação foram utilizados na semente provocaram redução na altura da planta desde a emergência. O CM pode ser utilizado no tratamento de sementes, tanto por embebição, quanto por aplicação direta, com efeitos semelhantes na redução da altura, da área foliar e da massa seca de folhas $e$ de caules das plantas de algodoeiro, com efeito proporcional à dose utilizada.

Palavras-chave: regulador de crescimento, métodos de tratamento, tratamento de sementes, Gossypium hirsutum r. latifolium.

\section{ABSTRACT}

The application of growth regulator through seed imbibitions has some drawbacks, including the requirement of drying the seeds after treatment. The objective of this research was to evaluate the effect of seeds treatment with Mepiquat
Chloride (MC) on cotton plant growth by direct application and soaking. The treatments were arranged in randomized blocks with six replicates, using PR 02-307 lineage. The experiment consisted of five treatments: untreated seeds; direct application of MC on the seeds with 3.75 and $7.5 \mathrm{~g}$ a.i. $\mathrm{kg}^{-1}$ of seed and soaking in solutions of 3.75 and $7.5 \mathrm{~g}$ a.i. $\mathrm{kg}^{-1}$ of seeds. After the treatments, the seeds were sowed in three liters vases in a greenhouse. Height and leaf area were evaluated until 90 days after emergency and dry matter weight was evaluated at end of the experiment. The treatments that used growth regulator on seeds led to plant height reduction from emergency, regardless of dose and form of application. MC can be used by seed soaking or direct application on the seeds, as both have similar effects on height reduction, leaf area, dry matter of leaves and stems of cotton plants. The effect is doseproportional.

Key words: plant growth regulator, treatment methods, seeds treatment, Gossypium hirsutum r. latifolium.

\section{INTRODUÇÃO}

O regulador de crescimento Cloreto de Mepiquat (CM) vem sendo utilizado há anos para o controle da altura de plantas do algodoeiro. Ele interfere na biossíntese do ácido giberélico, inibindo-a e reduzindo, assim, o crescimento em face da menor elongação celular (LAMAS, 2001). É utilizado em aplicações foliares, principalmente de forma parcelada, desde o estádio de botão floral até o florescimento.

Com o uso de CM, são obtidas plantas com alteração na partição da biomassa, inibindo o

\footnotetext{
'Departamento de Agronomia, Universidade Estadual de Londrina (UEL), CP 6001, 86051-990, Londrina, PR, Brasil. E-mail: gtnagashima@yahoo.com.br.*Autor para correspondência.

IIInstituto Agronômico do Paraná (IAPAR), Londrina, PR, Brasil.

IIIAutônomo, Luiz Eduardo Magalhães, BA, Brasil.
} 
crescimento de determinadas partes e estimulando outras, o que confere maior eficiência às plantas, em especial maior tolerância ao estresse hídrico (FERNÁNDEZ etal., 1991; COTHREN\& OOSTERHUIS, 1993). O ideal é que a relação entre a massa seca da parte reprodutiva e a vegetativa do algodoeiro seja maior que uma unidade, pois, nesse caso, a correlação com a produção é positiva (MEREDITH JR. \& WELL, 1989).

Após a aplicação foliar, a absorção do regulador é influenciada por diversos fatores do ambiente (ZHAO \& OOSTERHUIS, 1998), dentre eles, o intervalo sem chuvas após a sua aplicação. MATEUS et al. (2004) e SOUZA \& ROSOLEM (2007) relatam que quanto menor o intervalo entre a aplicação e a precipitação, maior a quantidade de produto a ser reposta. Segundo os autores, uma chuva de $10 \mathrm{~mm}$ até 16 horas após a aplicação é suficiente para lavar o regulador. Como alternativa para contornar o problema das eventuais perdas do produto aplicado via foliar causado pelas chuvas, o tratamento de sementes de algodão com CM vem sendo estudado. A aplicação do CM na semente também tem mostrado efeito positivo em condições de estresse fitotóxico no uso de herbicidas (CORBIN \& FRANS, 1991) e na promoção do enraizamento (DUAN et al., 2004).

Sementes da cultivar 'IPR 120' foram embebidas em soluções com diferentes concentrações do produto comercial $[0 ; 0,5 ; 2,5 ; 5,0 ; 7,5 \%(\mathrm{v} / \mathrm{v})]$ e três tempos de embebição (três, seis e 12 horas) por NAGASHIMA et al. (2005), constatando-se redução da fitomassa seca, da área foliar, da altura da inserção do primeiro nó cotiledonar e da altura das plantas desde a emergência. Os resultados indicaram a possibilidade de utilização dessa metodologia para a obtenção de plantas de menor estatura, o que auxiliaria na implantação de cultivos adensados do algodoeiro.

Em condições de campo, YEATES et al. (2005) compararam os tratamentos de sementes de algodão com regulador de crescimento via embebição e aplicação direta na semente e concluíram que ambas as formas de aplicação provocaram reduções precoces no porte das plantas. A redução do porte e a duração do efeito redutor foram sensíveis às concentrações utilizadas. Segundo os autores, houve atraso no desenvolvimento das plantas, e o rendimento da cultura foi reduzido em doses a partir de $4,0 \mathrm{~g} \mathrm{~kg}^{-1}$ de sementes de CM.

O regulador CM reduziu o crescimento das plantas, da emergência até o início do florescimento quando aplicado diretamente na semente juntamente com fungicidas (LAMAS, 2006).
Em condições de campo, com algodoeiro da cultivar 'IPR 120', oriundo de sementes embebidas em soluções de diversas concentrações de CM, NAGASHIMA et al. (2007) demonstraram que essa forma de aplicação pode provocar redução no porte da planta que persistiu até o início da formação do botão floral. Contudo, o efeito não afetou a produção de algodão em caroço.

Em outros experimentos (CORBIN \& FRANS, 1991; XU \& TAYLOR, 1992; DUAN et al., 2004), diferentemente dos experimentos de YEATES et al. (2005) e LAMAS (2006), o tratamento de sementes foi realizado por imersão da semente (embebição) em diversas concentrações e tempos de embebição. Entretanto, a adoção dessa metodologia de utilização, em extensas áreas de cultivo, pode ser dificultada pela necessidade de secagem das sementes embebidas. Assim, há necessidade de desenvolvimento de novos modos de aplicação do regulador de crescimento CM a fim de permitir que esse método possa ser empregado em condições de campo, atendendo as exigências demandadas pela cotonicultura.

O objetivo do presente trabalho foi o de avaliar o tratamento de sementes com CM, comparando os efeitos de dois métodos de aplicação, via embebição e aplicação direta na semente de algodão, e das doses utilizadas sobre o crescimento e desenvolvimento das plantas de algodoeiro.

\section{MATERIAL E MÉTODOS}

O experimento foi conduzido em condições de casa de vegetação, no Instituto Agronômico do Paraná (IAPAR), em Londrina, Paraná, região Sul do Brasil (2329'41,4”'S e 51¹2'5,5”'W). O delineamento experimental utilizado foi o blocos ao acaso, com cinco tratamentos e seis repetições, em esquema fatorial 2x2x1 (dois métodos de aplicação, duas doses e um tratamento testemunha), com os seguintes tratamentos: 1) T1: controle - sementes sem qualquer tratamento; 2 ) T2: métodos de aplicação direta na dose 1 de 3,75g i.a. $\mathrm{kg}^{-1}$ de sementes; 3) T3: método de aplicação direta na dose 2 de 7,5g i.a. $\mathrm{kg}^{-1}$ de sementes; 4) T4: método de sementes embebidas por $5 \mathrm{~h}$ na dose 1 de 3,75 g i.a. kg-1 de sementes e 5) T5: método de sementes embebidas por 5 horas na dose 2 de $7,5 \mathrm{~g}$ i.a. $\mathrm{kg}^{-1}$. Utilizou-se o produto comercial com 250 g i.a. $\mathrm{L}^{-1} \mathrm{deCM}$.

Para embebição de sementes, foram gastos 400mL da solução para cada kg de sementes, na temperatura de $25 \pm 0,5^{\circ} \mathrm{C}$. Pelo método da aplicação direta por aspersão, as sementes $(1 \mathrm{~kg})$ foram acondicionadas em sacos plásticos, sendo o produto regulador aplicado diretamente sobre estas, sendo 
utilizados 15 e $30 \mathrm{~mL}$ do produto comercial, contendo 250 g i.a. $\mathrm{L}^{-1}$ e correspondendo a 3,75 e $7,5 \mathrm{~g}$ i.a. $\mathrm{kg}^{-1} \mathrm{de}$ sementes, com agitação manual vigorosa até distribuição uniforme. As sementes de algodão utilizadas foram da linhagem PR 02-307 (IAPAR), com porte considerado médio para alto, arquitetura de planta com formato cilíndrico e ciclo médio de 150 dias para início da colheita, deslintadas quimicamente pelo método via úmida com o uso de ácido sulfúrico concentrado.

As sementes foram tratadas no dia 15 de janeiro de 2007, e a semeadura ocorreu quatro dias após, em vasos pretos de polipropileno, com capacidade de três litros, preenchidos com solo agricultável da região (latossolo vermelho distroférrico) misturado com 30\% do volume de areia. A emergência ocorreu no dia 24 de janeiro. Os vasos foram regados diariamente, e os tratamentos fitossanitários foram os recomendados para a cultura.

As alturas de plantas em centímetros foram avaliadas desde o nível do solo até o ápice da planta com uma régua escolar, aos 14, 35, 49 e 90DAE, área foliar por planta em $\mathrm{cm}^{2}$ foi avaliada pelo método não destrutivo (NAGASHIMA et al., 2005) aos 14, 35 e 90DAE.

No final do experimento, aos 90 dias da emergência, foram determinadas a fitomassa, em gramas, da matéria seca das folhas, considerada somente a lâmina foliar; do caule incluindo o pecíolo; da estrutura reprodutiva e da raiz, secas em estufa com ventilação forçada e temperatura de $55 \pm 2^{\circ} \mathrm{C}$ até a obtenção da massa constante.

Os dados obtidos foram submetidos à análise de variância (teste F), em nível de 5\% de significância.

\section{RESULTADOS E DISCUSSÃO}

Conforme se observa na tabela 1 , para a altura de plantas, todos os tratamentos apresentaram diferenças altamente significativas em relação ao tratamento testemunha, mesmo no término do experimento, aos 90DAE, comprovando que, independentemente dos tipos de aplicação e das doses utilizadas, houve efeito do regulador de crescimento, reduzindo o porte das plantas de algodão. Esses resultados estão de acordo com NAGASHIMA et al. (2005) e YEATES et al. (2005). O baixo porte das plantas controle verificado aos 90DAE deve-se ao fato de terem sido utilizados vasos com pouco volume e também de não ter sido efetuada a adubação em cobertura.

Não houve diferenças no efeito dos métodos de aplicação, durante todo o período do experimento. A aplicação direta na semente e a embebição de sementes com o CM apresentaram-se efetivas na redução do porte, sendo os efeitos obtidos concordantes com os dados obtidos por NAGASHIMA et al. (2005), que obtiveram redução no porte das plantas originadas de sementes embebidas com o CM e por LAMAS (2006), que utilizou a aspersão direta nas sementes, obtendo redução da altura das plantas até o início do florescimento.

O efeito das doses utilizadas foi significativo durante todo o período analisado. Esses resultados, em condições de cultivo em vaso e em casa de vegetação, estão de acordo com os obtidos por NAGASHIMA et al. (2005), que verificaram a maior redução da altura de plantas de algodão quando utilizadas doses maiores do regulador de crescimento CM.

Tabela 1 - Altura média de plantas de algodoeiro originadas de sementes sem tratamento (T1) e tratadas com CM por aplicação direta com $3,75 \mathrm{~g} \mathrm{~kg}^{-1}$ (T2) e 7,5g kg-1 (T3) e embebição com 3,75 $\mathrm{kg}^{-1}$ (T4) e 7,5g kg-1 (T5) aos 14, 35, 49 e 90 dias após emergência (DAE), e valores de F calculados, Londrina, 2007.

\begin{tabular}{|c|c|c|c|c|}
\hline \multirow{2}{*}{ Tratamentos } & \multicolumn{4}{|c|}{-Altura média (cm) } \\
\hline & 14DAE & 35DAE & 49DAE & 90DAE \\
\hline $\mathrm{T} 1$ & 26,05 & 43,17 & 49,50 & 51,58 \\
\hline $\mathrm{T} 2$ & 16,40 & 33,42 & 37,42 & 37,75 \\
\hline T3 & 12,37 & 24,67 & 28,08 & 28,67 \\
\hline $\mathrm{T} 4$ & 15,73 & 29,58 & 33,25 & 34,67 \\
\hline $\mathrm{T} 5$ & 12,65 & 26,08 & 29,17 & 30,42 \\
\hline T1 vs Fatores & $132,00^{*}$ & $\begin{array}{l}-----V a \\
43,16^{*}\end{array}$ & $43,42^{*}$ & $73,70^{*}$ \\
\hline Método aplicação (MA) & $0,04^{\mathrm{ns}}$ & $0,36^{\mathrm{ns}}$ & $0,42^{\mathrm{ns}}$ & $0,12^{\mathrm{ns}}$ \\
\hline Doses (D) & $15,10^{*}$ & $9,33^{*}$ & $7,96^{*}$ & $11,70^{*}$ \\
\hline (MA) vs (D) & $0,27^{\mathrm{ns}}$ & $1,71^{\mathrm{ns}}$ & $1,22^{\mathrm{ns}}$ & $1,54^{\mathrm{ns}}$ \\
\hline CV (\%) & 13,48 & 15,65 & 16,42 & 13,04 \\
\hline
\end{tabular}

* significativo em nível de 5\% de probabilidade, pelo teste F. 
Para a área foliar estimada, o tratamento testemunha apresentou diferença quando comparado com tratamentos com o regulador de crescimento, sendo observadas reduções significativas aos 14 e 35DAE (Tabela 2). Aos 90DAE, não foram observadas diferenças entre os tratamentos. A aplicação direta do regulador de crescimento na semente não apresentou diferença com a aplicação via embebição na semente. Comparando-se as doses utilizadas, o seu efeito manifestou-se até 35DAE. Houve interação entre os fatores analisados somente quando a da avaliação foi efetivada aos 35DAE, sendo o efeito da dose verificado dentro do método de aplicação do regulador de crescimento via aplicação direta, apresentando $420,8 \mathrm{~cm}^{2}$ quando utilizada a dose menor de 3,75g i.a. $\mathrm{kg}^{-1}$ de sementes e $321,0 \mathrm{~cm}^{2}$ para a dose de $7,5 \mathrm{~g}$ i.a. $\mathrm{kg}^{-1}$ de sementes. Quando utilizado o regulador via embebição de sementes, não foi notada diferença entre as doses. Esses resultados corroboram os obtidos por NAGASHIMA et al. (2005), que obtiveram redução da área foliar por planta, com a embebição de sementes com CM, e o efeito do tratamento perdurou até o aparecimento do botão floral do algodoeiro. STUART et al. (1984) utilizaram o regulador via aplicação foliar entre o estádio de botão floral e florescimento, obtendo redução no índice de área foliar pela redução no número de folhas e pelo tamanho da folha. Segundo FERNÁNDEZ et al. (1991), o CM inibe a expansão foliar e, consequentemente, origina plantas com arquitetura mais compacta.
A fitomassa da matéria seca de folhas avaliada no final do experimento, aos 90DAE, não apresentou diferença quando comparada entre os tratamentos e a testemunha. No entanto, quando comparado o efeito do regulador de crescimento nos métodos de aplicação utilizados, houve diferença, com a embebição apresentando menor efeito redutor. A fitomassa do caule apresentou diferenças entre os tratamentos e a testemunha, com diferença entre as doses quando utilizada a aplicação direta do regulador CM na semente, com redução da fitomassa com o uso de dose maior. Para os fatores fitomassa de raízes e estruturas reprodutivas, não houve diferença alguma entre os tratamentos e entre diferentes métodos da aplicação do regulador e também nas doses utilizadas (Tabela 2). Esses resultados corroboram os obtidos por NAGASHIMA et al. (2005/2007) em condições de casa de vegetação e cultivo em campo.

Com os resultados obtidos neste experimento, é possível recomendar a utilização do tratamento de sementes de algodão com o uso da aplicação direta de CM, pois as respostas foram similares aos da embebição do produto pelas sementes; entretanto, há necessidade de maiores estudos em condições de campo.

\section{CONCLUSÕES}

$O$ regulador de crescimento $\mathrm{CM}$ nas sementes reduz a altura da planta de algodão, a área

Tabela 2 - Médias da área foliar aos 14, 35 e 90 dias após emergência (DAE) e da fitomassa da matéria seca de folhas, caules e raízes e de estruturas reprodutivas aos 90 dias após emergência originada de sementes sem tratamento (T1) e tratadas com CM pelo método de aplicação via direta (MA1) com 3,75 $\mathrm{kg}^{-1}$ (T2) e 7,5g kg-1 (T3) e método de aplicação via embebição (MA2) com 3,75g kg-1 (T4) e 7,5g kg-1 (T5) e valores de F calculados, Londrina, 2007.

\begin{tabular}{|c|c|c|c|c|c|c|c|}
\hline \multirow[b]{2}{*}{ Tratamentos } & \multicolumn{3}{|c|}{--------Área foliar estimada $\left(\mathrm{cm}^{2}\right)$-------- } & \multicolumn{4}{|c|}{---------Fitomassa da matéria seca $\left(\right.$ g planta $^{-1}$ )------- } \\
\hline & $14 \mathrm{DAE}$ & $35 \mathrm{DAE}$ & $90 \mathrm{DAE}$ & Folhas & Caules & Raízes & Est. Rep. \\
\hline $\mathrm{T} 1$ & 142,8 & 509,8 & 550,1 & 2,63 & 3,96 & 1,73 & 7,17 \\
\hline $\mathrm{T} 2$ & 82,4 & 420,8 & 513,4 & 2,98 & 3,71 & 1,91 & 6,44 \\
\hline T3 & 62,5 & 321,0 & 449,9 & 2,38 & 2,60 & 1,77 & 6,36 \\
\hline $\mathrm{T} 4$ & 80,8 & 355,0 & 493,8 & 3,03 & 3,66 & 2,06 & 6,00 \\
\hline $\mathrm{T} 5$ & 72,2 & 391,8 & 535,3 & 3,49 & 3,15 & 2,17 & 5,67 \\
\hline T1 vs Fatores & $98,84^{*}$ & $15,86^{*}$ & $1,35^{\text {ns }}$ & $\begin{array}{c}1,42^{\text {ns }} \\
\end{array}$ & $4,49^{*}$ & $1,51^{\mathrm{ns}}$ & $2,53^{\mathrm{ns}}$ \\
\hline Método Aplicação (MA) & $0,43^{\mathrm{ns}}$ & $0,01^{\mathrm{ns}}$ & $0,68^{\mathrm{ns}}$ & $4,82^{*}$ & $0,72^{\mathrm{ns}}$ & $2,46^{\mathrm{ns}}$ & $0,92^{\mathrm{ns}}$ \\
\hline Doses (D) & $5,36^{*}$ & $1,04^{*}$ & $0,08^{\mathrm{ns}}$ & $0,17^{\mathrm{ns}}$ & $7,88^{*}$ & $0,01^{\mathrm{ns}}$ & $0,12^{\mathrm{ns}}$ \\
\hline$(\mathrm{MA}) \mathrm{vs}(\mathrm{D})$ & $0,84^{\mathrm{ns}}$ & $4,88^{\mathrm{ns}}$ & $1,72^{\mathrm{ns}}$ & $4,09^{\mathrm{ns}}$ & $1,09^{\mathrm{ns}}$ & $0,49^{\mathrm{ns}}$ & $0,04^{\mathrm{ns}}$ \\
\hline (D) dentro de (MA1) & & $5,21^{*}$ & & & & & \\
\hline (D) dentro de (MA2) & & $0,71^{\mathrm{ns}}$ & & & & & \\
\hline CV $(\%)$ & 17,06 & 18,95 & 19,26 & 21,14 & 20,69 & 22,62 & 23,03 \\
\hline
\end{tabular}

*significativo $\mathrm{e}^{\mathrm{ns}}$ : não significativo em nível de 5\% de probabilidade, pelo teste $\mathrm{F}$.

MA1: método aplicação via direta (T2 e T3); MA2: método aplicação via embebição (T4 e T5). 
foliar e a fitomassa da matéria seca de caule. Pode-se utilizar o CM via sementes por meio da embebição e também da aplicação direta.

As doses utilizadas foram efetivas para a redução da altura de plantas de algodão.

\section{AGRADECIMENTO}

À Coordenação de Aperfeiçoamento de Pessoal de Nível Superior (CAPES), pela concessão de bolsa ao primeiro autor.

\section{REFERÊNCIAS}

CORBIN, B.R.; FRANS, R.E. Protecting cotton (Gossypium hirsutum) from Fluometuron injury with seed protectants. Weed Science, v.39, n.3, p.408-411, 1991.

COTHREN, J.T.; OOSTERHUIS, D.M. Physiological impact of plant growth regulators in cotton. In: BELTWIDE COTTON PRODUCTION RESEARCH CONFERENCES, 1993, Dallas, Texas. Proceedings...Memphis: National Cotton Council, 1993. p.128-132.

DUAN, L.et al. Promoting effects of mepiquat chloride on lateral roots initiation of cotton seedlings. Research Centre for Crop Chemical Regulation. Disponível em: <http:// www.pgrsa.org/Charleston_PGRSA_Proceedings_2004/papers/ 055.pdf>. Acesso em: 24 dez. 2007.

FERNÁNDEZ, C.J. et al. Partitioning of biomass in wellwatered and water-stressed cotton plants treated with mepiquat chloride. Crop Science, v.31, n.5, p.1224-1228, 1991.

LAMAS, F.M. Estudo comparativo entre cloreto de mepiquat e cloreto de chlormequat aplicados em algodoeiro. Pesquisa Agropecuária Brasileira, v.36, n.2, p.265-272, 2001. Disponível em: <http://www.scielo.br/pdf/\%0D/pab/v36n2/ a08v36n2.pdf>. Acesso em 24 jul. 2008. doi: 10.1590/S0100204X2001000200008.

LAMAS, F.M. Cloreto de mepiquat na cultura do algodoeiro via sementes. Dourados: EMBRAPA AGROPECUÁRIA OESTE, 2006. 19p. (Boletim de Pesquisa e Desenvolvimento 33). Disponível em: <http:// w w w . c p a o.e m b r a p a b r / p u b l i c a c o e s/ ficha.php?tipo=BP\&num=33\&ano=2006> . Acesso em 03 jan. 2008.
MATEUS, P.M. et al. Perdas de cloreto de mepiquat no algodoeiro por chuva simulada. Pesquisa Agropecuária Brasileira, v.39, n.7, p.631-636, 2004. Disponível em: <http:/ /scielo.br/scielo.php?script=sci_arttext \&pid=S0100204 $\mathrm{X} 2004000700003 \& \operatorname{lng}=\mathrm{en} \& \mathrm{nrm}=\mathrm{en} \& \mathrm{nrm}=\mathrm{iso}>$. Acesso em: 03 jan. 2008. doi: 10.1590/S0100-204X2004000700003.

MEREDITH JR, W.R.; WELL, R. Potential for increasing cotton yields through enhanced partitioning to reproductive structures. Crop Science, v.29, n.3, p.636-639, 1989.

NAGASHIMA, G.T. et al. Desenvolvimento de plantas de algodão provenientes de sementes embebidas com cloreto de mepiquat. Pesquisa Agropecuária Brasileira, v. 40, n.9, p. 943-946, 2005. Disponível em: <http://www.scielo.br/pdf/pab/ v40n9/a15v40n9.pdf>. Acesso em: 03 jan. 2008. doi: 10.1590/ S0100-204X2005000900015.

NAGASHIMA, G.T. et al. Embebição de sementes e aplicação foliar com cloreto de mepiquat no crescimento e produção do algodoeiro. Ciência e Agrotecnologia, v.31, n.4, p.10271034, 2007. Disponível em: < http://www.scielo.br/pdf/cagro/ v31n4/13.pdf >. Acesso em: 03 jan. 2008. doi: 10.1590/ S1413-70542007000400013.

SOUZA, F.S.; ROSOLEM, C.A. Rainfall intensity and mepiquat chloride persistence in cotton. Scientia Agricola, v.64, n.2, p.125130, 2007. Disponível em: <http://www.scielo.br/ scielo.php?pid=S0103-90162007000200004\&script=sci_arttext $>$. Acesso em: 05 fev. 2008. doi: 10.1590/S0103-90162007000200004.

STUART, B.L. et al. Modification of cotton water relations and growth with mepiquat chloride. Agronomy Journal, v.76, p.651-655, 1984.

$\mathrm{XU}, \mathrm{X}$; TAYLOR, H.M. Increase in drought resistance of cotton seedlings treated with mepiquat chloride. Agronomy Journal, v.84, p.569-574, 1992.

YEATES, S.J. et al. Cotton growth and yield after seed treatment with mepiquat chloride in the tropical winter season. Field Crops Research, n.93, p.122-131, 2005.

ZHAO, D.; OOSTERHUIS, D. Evaluation of plant growth regulators for effect of the growth and yield of cotton. In: BELTWIDE COTTON CONFERENCE, 1998, San Diego, CA. Proceedings... Memphis: National Cotton Council of America, 1998. p.1482-1484. 